\title{
Diversity of Blastocystis subtypes in dogs in different geographical settings
}

Wenqi Wang $^{1 *}$, Leigh Cuttell ${ }^{1}$, Helle Bielefeldt-Ohmann ${ }^{1,2}$, Tawin Inpankaew ${ }^{3,4}$, Helen Owen ${ }^{1}$ and Rebecca J Traub $b^{1,2}$

\begin{abstract}
Background: Blastocystis is a ubiquitous, globally distributed intestinal protist infecting humans and a wide range of animals. Several studies have shown that Blastocystis is a potentially zoonotic parasite. A 1996 study reported a 70\% Blastocystis prevalence in Brisbane pound dogs while another study found that pet dogs/cats of 11 symptomatic Blastocystis infected patients harboured at least one Blastocystis subtype (ST) in common with the patient. These results raised the possibility that dogs might be natural hosts of Blastocystis. In this study, we aimed to investigate this hypothesis by estimating the prevalence of Blastocystis carriage and characterising the diversity of STs in dogs from three different environmental settings and comparing these STs with the range that humans harbour.
\end{abstract}

Methods: Two hundred and forty faecal samples from dogs from three different geographical regions with varying levels of socio-economic development and sanitation, namely i) 80 pet and pound dogs from Brisbane, Australia, ii) 80 semi-domesticated dogs from Dong Village, Cambodia and iii) 80 stray dogs from the densely populated cities of Sikkim, Delhi and Mumbai in India, were screened for Blastocystis using PCR and subtyped based on the "barcode region" of the small subunit ribosomal RNA (SSU rRNA) gene.

Results: The prevalence of Blastocystis in dogs from Brisbane and Cambodia was 2.5\% (2/80) and 1.3\% (1/80), respectively, in contrast to $24 \%(19 / 80)$ in stray dogs from India. Stray dogs in India carried a diverse range of Blastocystis STs including ST 1, 4, 5 and 6 while the dogs from Brisbane carried only ST1 and one Cambodian dog carried ST2.

Conclusion: The results suggest there is geographical variation in Blastocystis prevalence and STs between dog populations as reported in human studies. In addition, the greater diversity of STs and higher prevalence of Blastocystis in Indian stray dogs compared to pet/pound and community dogs in Australia and Cambodia could reflect close proximity to humans and other animals and exposure to their faeces. It appears that dogs are not natural hosts for Blastocystis but rather are transiently and opportunistically infected with a diversity of STs.

Keywords: Blastocystis, Dog, Zoonosis, Epidemiology

\section{Background}

Blastocystis is a ubiquitous, intestinal protist with a high prevalence worldwide in humans and animals. Host types shown to carry Blastocystis include humans, non-human primates, a range of domesticated and wild mammals and birds [1]. Blastocystis is the most common gastrointestinal parasite recovered in human fecal parasite surveys, with prevalence ranging from $0.5 \%$ in developed countries to $60 \%$ in developing countries [2]. The most accepted

\footnotetext{
*Correspondence: w.wang3@uq.edu.au

'School of Veterinary Science, The University of Queensland Gatton Campus, Queensland 4343, Australia

Full list of author information is available at the end of the article
}

proposed mode of transmission is the fecal-oral route either by direct contact or food and water-borne transmission [3-5]. Molecular analysis of the SSU-rRNA gene has allowed for subdivision of Blastocystis into 14 distinct subtypes (STs) in humans, non-human primates (NHPs), mammals and birds $[1,6,7]$. Humans have been shown to carry STs 1-9, with ST3 being the most prevalent followed by ST1 [8].

Blastocystis is a potential zoonosis as suggested by a number of studies that have isolated identical STs of Blastocystis from humans and their in-contact animals [3-6,9]. Recently, domestic dogs were proposed as a

\section{Biomed Central}

(c) 2013 Wang et al.; licensee BioMed Central Ltd. This is an Open Access article distributed under the terms of the Creative Commons Attribution License (http://creativecommons.org/licenses/by/2.0), which permits unrestricted use, distribution, and reproduction in any medium, provided the original work is properly cited. 
potential source of Blastocystis infection to humans [10]. Eleven symptomatic patients, their pets, as well as $59 \%$ of family members tested positive for Blastocystis by PCR and all infected family members and domestic animals (dogs and cats) harboured at least one Blastocystis ST in common with the patient. A study by Duda et al. [11] on Blastocystis prevalence in 72 domestic dogs (70 pound dogs and 2 pet dogs) in Brisbane showed a $70.8 \%$ prevalence using light microscopy on fecal wet mounts. These results raised the possibility that dogs might be natural hosts of Blastocystis and potential sources of zoonotic transmission to humans. In this study, we aimed to investigate this hypothesis by estimating the prevalence of Blastocystis infection and characterising the diversity of STs in dogs from three different environmental settings and comparing these STs with the known range harboured by humans.

\section{Methods}

\section{Sampling}

A total of 240 dogs were screened for Blastocystis, including 80 dogs from each of the 3 following settings 1 ) pound and pet dogs in Brisbane, a major metropolitan area in Queensland, Australia 2) semi-domesticated dogs from 36 households in Dong Village, Cambodia, 3) stray street dwelling dogs from the Indian cities of Delhi, Sikkim and Mumbai. The three settings differed from each other in terms of geographical location, level of hygiene and opportunities for dogs to come into contact with faeces from humans and other animals.

In Brisbane, freshly voided faecal samples were collected off the ground from pet and pound dogs. These samples were obtained in 2010 - 2011 from 45 pound dogs and 35 pet dogs. Samples were stored at room temperature until DNA extraction was performed, usually within 12 hours. In Cambodia, faecal samples were collected per-rectum from 80 semi-domesticated dogs from 36 households in the Dong village and preserved in $2.5 \%$ potassium dichromate $\left(\mathrm{K}_{2} \mathrm{Cr}_{2} \mathrm{O}_{7}\right)$ (Sigma-Aldrich, Australia) until DNA extraction was performed. In India, stray dogs were sampled from three cities, namely Sikkim $(\mathrm{n}=25)$, Delhi $(\mathrm{n}=27)$ and Mumbai $(\mathrm{n}=28)$ in 2008 as part of a street dog sterilization project run by NonGovernmental Organisations and municipalities. Faecal samples were collected from stray dogs per-rectum and preserved in $70 \%$ ethanol. This project was approved by the University of Queensland Animal Ethics Committee with approval no. ANRFA/472/11.

\section{Molecular analysis DNA extraction}

DNA was extracted from faeces using the QIAamp DNA Stool Mini Kit (Qiagen, Germany) with minor modifications. Following addition of buffer ASL and homogenisation, samples were subjected to freeze/thaw 3 times repeatedly in liquid nitrogen and $95^{\circ} \mathrm{C}$ water, followed by a further 5 mins incubation at $95^{\circ} \mathrm{C}$ to lyse cells.

\section{Control testing of extracted DNA}

For internal process control, all samples were tested using published universal primers that amplify a $140 \mathrm{bp}$ fragment of the $18 \mathrm{~S}$ ribosomal RNA gene from eukaryotic DNA to detect for amplifiable DNA [12]. The primers used were forward: 18SEUDIR 5'-TCTGCCCTATCAA CTTTCGATGG-3' and reverse: 18SEUINV 5'-TAATT TGCGCGCCTGCTG-3'. PCR cycling conditions were optimised by modifying the published real-time PCR protocol using an annealing temperature of $60^{\circ} \mathrm{C}$. This testing was performed to check for inhibition and also ensure that the variation in the sample preservation methods would not affect the accuracy of results.

\section{PCR amplification}

Two previously published PCR primer sets and conditions were utilised for the detection and characterisation of Blastocystis STs [13-15]. A single step [14] and nested PCR [13,15] were performed on each sample using a Bio-Rad C1000 Thermal Cycler (Bio-Rad Laboratories, Inc., Hercules, USA) (Table 1) to amplify a $600 \mathrm{bp}$ and $1100 \mathrm{bp}$ region of the SSU rRNA gene, respectively. Details of primer sets and PCR cycling conditions are outlined in Table 1.

\section{Phylogenetic analysis}

PCR products were purified using the PureLink Genomic DNA Mini Kit (Life Technologies Corporation, New York, USA) according to the manufacturer's protocol. Unidirectional DNA sequencing was carried out using the respective reverse primers with an Applied Biosystems 3130/ 3130xl Genetic Analyzer. DNA sequences were analysed using Finch TV v 1.4.0 (Geospiza Inc., Seattle, WA, USA) and compared with previously published sequences from GenBank (National Center for Biotechnology Information) using Basic Local Alignment Search Tool (BLAST) 2.2.9 [16]. The sequences were aligned with previously published sequences of the SSU rRNA gene of the various Blastocystis STs sourced from GenBank using BioEdit v 7.1.3.0 software (Ibis Biosciences, Carlsbad, CA, USA). Neighbour joining analysis and construction of a tree was carried out using Mega 4.1 software (The Biodesign Institute, Tempe, AZ, USA). Proteromonas lacerate (U37108) was used as an out-group.

\section{Statistical analysis}

Prevalence and their 95\% confidence intervals for each group of dogs were calculated using EpiTools epidemiological calculators [17]. 
Table 1 PCR primer sets for amplification of SSU rRNA region of Blastocystis from dog faeces

\begin{tabular}{lll}
\hline Primer & Primer name and sequence (5' to $\mathbf{3}^{\prime}$ ) & Product size \\
\hline Single step PCR conditions as per Scicluna et al. [14] & BhRDr -GAGCTITTTAACTGCAACAACG & 600 bp \\
& RD5 -ATCTGGTTGATCCTGCCAGT \\
Nested PCR-primary set, conditions as per Clark [13] & RD3-GGGATCCTGATCCTTCCGCAGGTCACCTAC \\
& RD5 -GGAAGCTTATCTGGTTGATCCTGCCAGTA \\
Nested PCR - secondary set, conditions as per Bohm-Gloning [15], & Forward -GGAGGTAGTGACAATAAATC \\
using 1 ul of PCR product from primary step. & Reverse - CGTTCATGATGAACAATTAC \\
\hline
\end{tabular}

\section{Results and discussion}

Summary of PCR results are shown in Tables 2 and 3. Of the 80 Indian dogs tested, 19 (24\%; 95\% CI, 14.4, 33.1) were positive for Blastocystis with a predominance of ST1 and ST6. The Blastocystis prevalence in the cities of Sikkim, Mumbai and Delhi were 24\% (6/25), 14\% $(4 / 28)$ and $33 \%(9 / 27)$ respectively, with dogs in all cities harbouring ST 1, 4, 5 or 6 . Of the 80 Brisbane dogs, $2(2.5 \%, 95 \%$ CI, 0, 5.9) samples were positive, both were from pound dogs and were ST1. Of the 80 Cambodian dogs, 1 (1.3\%, 95\% CI, 0, 3.7) was positive for ST2. The Brisbane prevalence results of our study are in contrast to those of Duda et al. [11] who found, using light microscopy only, that $70.8 \%$ of pound dogs from Brisbane harbored Blastocystis, a much higher prevalence than the $2.5 \%$ described here. This discrepancy could be attributed to the level of care and hygiene of the pound dogs in 1998 compared to current standards, while the source of acquisition and duration of stay within the pound or other confounding factors could have influenced Blastocystis prevalence. On the other hand, Blastocystis is a morphologically pleomorphic organism and the extensive variation in the appearance of the recognized forms of Blastocystis may lead to operator misinterpretations $[2,18,19]$ and in this case, potentially, a high rate of false positives.

Blastocystis prevalence can vary between countries and subpopulations, however, the general trend in previous prevalence studies is that developing countries have a

Table 2 PCR results of dog faecal samples

\begin{tabular}{|c|c|c|c|}
\hline & Positive & $\begin{array}{l}\text { Blastocystis } \\
\text { STs }\end{array}$ & Negative \\
\hline \multirow{4}{*}{$\begin{array}{l}\text { Indian street dogs (80) } \\
\text { (preserved in ethanol) }\end{array}$} & \multirow[t]{4}{*}{19 (24\%) } & ST1 - 9 & \multirow[t]{4}{*}{61 (76\%) } \\
\hline & & ST6 - 7 & \\
\hline & & ST $4-2$ & \\
\hline & & ST5 - 1 & \\
\hline $\begin{array}{l}\text { Brisbane dogs (80) } \\
\text { (fresh samples) }\end{array}$ & $\begin{array}{l}2(2.5 \% \\
\text { pound dogs) }\end{array}$ & ST1 - 2 & 78 (97.5\%) \\
\hline $\begin{array}{l}\text { Cambodian dogs (80) } \\
\text { (preserved in } 2.5 \% \mathrm{~K}_{2} \mathrm{Cr}_{2} \mathrm{O}_{7} \text { ) }\end{array}$ & 1 (1.3\%) & ST2 - 1 & 79 (98.7\%) \\
\hline
\end{tabular}

higher prevalence than that of developed countries [2]. Prevalence can range from as low as $3.3 \%$ in human samples from hospitals in Singapore [20] to $49 \%$ in samples from a Nigerian clinic and $70 \%$ in children in three counties in Liberia as reported in a ST distribution study by Alfellani et al. [8]. Both studies used PCR for diagnosis. In humans, these differences can be attributed to poorer sanitation, higher risk of food and water contamination and also greater exposure to animals and their excrements in developing countries as compared to the developed countries [2,4,21]. Our study, however, did not clearly discriminate between the prevalence of Blastocystis between dogs living in developing and developed communities. Mumbai, Delhi and Sikkim have been described as densely populated cities in India with inadequate toileting facilities and sewage systems leaving large sectors of these cities with poor sanitation and hygiene especially in the slum areas [22,23]. As a result, it is estimated that greater than $50 \%$ of resource-poor communities within urban areas practice open defecation [23,24]. This may explain the discrepancy between the prevalence of Blastocystis in the Indian stray dogs as compared to the Brisbane dogs, however, it does not account for the negligible prevalence of Blastocystis in semi-domesticated community dogs in rural Cambodia.

The Indian stray dogs in this study harbored ST 1, 4, 5 and 6 while the Brisbane and Cambodian dogs harbored only ST1 and ST2, respectively. Recently, Alfellani et al. $[8,25]$ consolidated the results of multiple studies on Blastocystis ST distribution in humans and NHPs in

Table 3 PCR results of dog faecal samples based on individual primer sets

\begin{tabular}{cccc}
\hline PCR & $\begin{array}{c}\text { Indian street } \\
\text { dogs }\end{array}$ & $\begin{array}{c}\text { Brisbane } \\
\text { dogs }\end{array}$ & Cambodian dogs \\
\hline \multirow{4}{*}{$\begin{array}{c}\text { Single step } \\
\text { PCR }\end{array}$} & ST1 -8 & ST1 - & ST2 -1 \\
& ST -2 & & \\
Nested PCR $5-1$ & & \\
& ST6 -2 & & ST2 - 1 \\
& ST1 -1 & All negative & (same sample as above) \\
\hline
\end{tabular}


several countries. The most common human STs in the UK for example, were ST3 and 4, while in Africa it was ST1 and 3 [8]. In the NHP study, they found that distribution of ST1, 2 and 3 appeared to be independent of geographical association or NHP group, whereas ST 8 was only observed in arboreal NHPs and species native to Asia or South America [25]. Collectively the results show variable geographical distribution of Blastocystis STs in both humans and NHPs between and within subpopulations of different countries. This hypothesis of geographical variation of STs might, at least in part, explain the results in this study, where we found different STs in dogs in different geographical regions except for ST1 which was found in both Indian and Brisbane dogs.

The general low prevalence of Blastocystis and the diversity of STs found in dogs in three different geographical regions/ settings suggest that dogs may be transiently and opportunistically infected by whichever Blastocystis ST is present in their environment, be it from a human or non-human source. Therefore, dogs are unlikely to act as either natural hosts or primary zoonotic reservoirs for Blastocystis but are capable of shedding potentially zoonotic STs and may therefore act as secondary zoonotic reservoirs for infection. The Blastocystis STs observed in dogs in this study include STs 1, 2, 4, 5 and 6. ST1 is one of the most common human STs, ST2 is common in UK, Brazil and Central Asia, while ST4 is very common in humans in the UK but rare in other countries [8], though rodents have been shown to be the main animal reservoir of ST4 [1]. ST5 is rare in humans but otherwise commonly reported in ungulates (e.g. pigs, cattle), whereas ST6 is uncommon in humans but otherwise reported mainly in avian species $[1,9,26]$. Keeping this in mind and the fact that coprophagia is common practice in dogs, the greater prevalence and diversity of STs found in the Indian stray dogs could be attributed to higher population density and greater exposure in their environment to fecal material from human and non-human hosts (cattle, pigs, avians, NHPs), from which they could have either mechanically passaged or acquired opportunistic infections with various Blastocystis STs. Ideally, studies to ascertain the relative prevalence of Blastocystis STs in humans and animals residing in these three geographical regions especially that of the urban centres in India would provide a clearer epidemiological picture of transmission pathways. Additionally, given that extensive genetic diversity exists within Blastocystis STs, ideally future molecular characterisation and comparison of dog, human and other mammalian Blastocystis STs using multilocus sequence typing (MLST) performed within communities endemic for Blastocystis in dogs and humans will shed further light on their role as natural hosts for infection [27].

\section{Conclusions}

Our results show that a diverse range of Blastocystis STs (ST1, 2, 4,5 and 6) can be found in dogs and it is likely that there is geographical variation of STs in dogs as has been shown in humans and NHPs $[8,25]$. Secondly, considering the low prevalence of Blastocystis in dogs with no indication of dog-specific/predominant ST, they are unlikely to be natural hosts of Blastocystis and are potentially opportunistically infected via coprophagia of other hosts faeces or contaminated drinking water. Larger scale Blastocystis epidemiological studies on humans and dogs from the same geographical areas with SSU rDNA (18S) allele analysis or MLST would be required to confirm this hypothesis.

\section{Competing interests}

The authors declare that they have no competing interests.

\section{Authors' contributions}

Conceived and designed the experiments: WW, LC, HBO, HO, RT. Sample collection: WW, TI, RT. Performed the experiments: WW, LC, TI. Analysed the data: WW, LC, HBO, TI, HO, RT. Wrote the manuscript: WW, LC, HBO, HO, RT. All authors read and approved the final manuscript.

\section{Acknowledgments}

This project has been supported by the William Peter Richards Trust Award Grant (WW) and The University of Queensland New Staff Start-up Grant (HO). Special thanks to the staff in the School of Veterinary Science, the UQ Veterinary Clinical Studies Centre and Logan City Council for their assistance in sample collection and processing of the Brisbane dog samples. Samples obtained from India were supplied by a Bayer Animal Health funded project carried out by Dr. Puteri Azaziah Megat Abd Rani as part of her PhD project with kind assistance from Vets Beyond Borders, Jeevasharam, Krishanasharam and In Defence of Animals, India. The Cambodian dog samples were collected as part of Dr. Tawin Inpankaew's PhD project that is funded by the University of Copenhagen, Denmark. We would also like to acknowledge staff of The National Center for Parasitology, Entomology and Malaria Control, Cambodia, Mr. Wissanuwat Chimnoi and Ms. Supanun Boonchob, staff of The faculty of Veterinary Medicine, Kasetsart University, Thailand, Dr. Chhay Somany, deputy director of the provincial health department at Preah Vihear province and all the participants for their assistance in sample collection from Dong village in Cambodia.

\section{Author details}

${ }^{1}$ School of Veterinary Science, The University of Queensland Gatton Campus, Queensland 4343, Australia. ${ }^{2}$ Australian Infectious Disease Research Centre, The University of Queensland, Queensland, Australia. ${ }^{3}$ Department of Veterinary Disease Biology, Faculty of Health and Medical Science, University of Copenhagen, Copenhagen, Denmark. ${ }^{4}$ Department of Parasitology, Faculty of Veterinary Medicine, Kasetsart University, Bangkok, Thailand.

Received: 3 June 2013 Accepted: 22 July 2013

Published: 24 July 2013

\section{References}

1. Stensvold CR, Alfellani MA, Norskov-Lauritsen S, Prip K, Victory EL, Maddox C, Nielsen HV, Clark CG: Subtype distribution of Blastocystis isolates from synanthropic and zoo animals and identification of a new subtype. Int J Parasitol 2009, 39(4):473-479.

2. Tan KS: New insights on classification, identification, and clinical relevance of Blastocystis spp. Clin Microbiol Rev 2008, 21(4):639-665.

3. Leelayoova S, Rangsin R, Taamasri P, Naaglor T, Thathaisong U, Mungthin M: Evidence of waterborne transmission of Blastocystis hominis. AmJTrop Med Hyg 2004, 70(6):658-662.

4. Leelayoova S, Siripattanapipong S, Thathaisong U, Naaglor T, Taamasri P, Piyaraj $P$, Mungthin M: Drinking water: a possible source of Blastocystis 
spp. subtype 1 infection in schoolchildren of a rural community in central Thailand. AmJTrop Med Hyg 2008, 79(3):401-406.

5. LI L, Tan TC, Tan PC, Nanthiney DR, Biraj MK, Surendra KM, Suresh KG: Predominance of Blastocystis sp. subtype 4 in rural communities, Nepal. Parasitol Res 2012, 110(4):1553-1562.

6. Parkar U, Traub RJ, Vitali S, Elliot A, Levecke B, Robertson I, Geurden T, Steele J, Drake B, Thompson RC: Molecular characterization of Blastocystis isolates from zoo animals and their animal-keepers. Vet Parasitol 2010, 169(1-2):8-17.

7. Fayer $\mathrm{R}$, Santin $\mathrm{M}$, Macarisin D: Detection of concurrent infection of dairy cattle with Blastocystis, Cryptosporidium, Giardia, and Enterocytozoon by molecular and microscopic methods. Parasitol Res 2012, 111(3):1349-1355.

8. Alfellani MA, Stensvold CR, Vidal-Lapiedra A, Onuoha ES, Fagbenro-Beyioku AF, Clark CG: Variable geographic distribution of Blastocystis subtypes and its potential implications. Acta Trop 2013, 126(1):11-18.

9. Yan Y, Su S, Ye J, Lai X, Lai R, Liao H, Chen G, Zhang R, Hou Z, Luo X: Blastocystis sp. subtype 5: a possibly zoonotic genotype. Parasitol Res 2007, 101(6):1527-1532.

10. Nagel R, Cuttell L, Stensvold CR, Mills PC, Bielefeldt-Ohmann H, Traub RJ. Blastocystis subtypes in symptomatic and asymptomatic family members and pets and response to therapy. Intern Med J 2012, 42(11):1187-1195.

11. Duda A, Stenzel DJ, Boreham PF: Detection of Blastocystis sp. in domestic dogs and cats. Vet Parasitol 1998, 76(1-2):9-17.

12. Fajardo V, Gonzalez I, Martin I, Rojas M, Hernandez PE, Garcia T, Martin R: Real-time PCR for detection and quantification of red deer (Cervus elaphus), fallow deer (Dama dama), and roe deer (Capreolus capreolus) in meat mixtures. Meat Sci 2008, 79(2):289-298.

13. Clark CG: Extensive genetic diversity in Blastocystis hominis. Mol Biochem Parasitol 1997, 87(1):79-83.

14. Scicluna SM, Tawari B, Clark CG: DNA barcoding of Blastocystis. Protist 2006, 157(1):77-85.

15. Bohm-Gloning B, Knobloch J, Walderich B: Five subgroups of Blastocystis hominis from symptomatic and asymptomatic patients revealed by restriction site analysis of PCR-amplified 16S-like rDNA. Trop Med Int Health 1997, 2(8):771-778.

16. National Library of Medicine: Basic Local Alignment Search Tool (BLAST) 2.2.9. http://blast.ncbi.nlm.nih.gov/Blast.cgi.

17. AusVet Animal Health Services and Australian Biosecurity Cooperative Research Centre for Emerging Infectious Disease: Epitools epidemiological calculators. http://epitools.ausvet.com.au.

18. Utzinger J, Botero-Kleiven S, Castelli F, Chiodini PL, Edwards H, Kohler N, Gulletta M, Lebbad M, Manser M, Matthys B, et al: Microscopic diagnosis of sodium acetate-acetic acid-formalin-fixed stool samples for helminths and intestinal protozoa: a comparison among European reference laboratories. Clin Microbiol Infec 2010, 16(3):267-273.

19. Stensvold CR, Arendrup MC, Jespersgaard C, Molbak K, Nielsen HV: Detecting Blastocystis using parasitologic and DNA-based methods: a comparative study. Diagn Micr Infec Dis 2007, 59(3):303-307.

20. Wong KH, Ng GC, Lin RT, Yoshikawa H, Taylor MB, Tan KS: Predominance of subtype 3 among Blastocystis isolates from a major hospital in Singapore. Parasitol Res 2008, 102(4):663-670.

21. Lee LI, Chye TT, Karmacharya BM, Govind SK: Blastocystis sp.: waterborne zoonotic organism, a possibility? Parasit Vectors 2012, 5:130-134

22. India Water Portal: National Urban Sanitation Policy: Towards City Wide Sanitation. http://www.indiawaterportal.org/sites/indiawaterportal.org/files/ National\%20Urban\%20Sanitation\%20Policy_Towards\%20city\%20wide\% 20sanitation_Consultation\%20workshop\%20on\%20city\%20sanitation\% 20plans_GTZ-ASEM_Nivedita\%20E.P._2010.pdf.

23. The United Nations for Children's Fund (UNICEF) (India): Water, Environment, Sanitation. http://www.unicef.org/india/wes.html.

24. World Health Organisation (WHO): WHO/UNICEF joint monitoring report 2012 Progress on drinking water and sanitation. http://www.who.int/ water_sanitation_health/monitoring/jmp2012/fast_facts/en/index.html.

25. Alfellani MA, Jacob AS, Perea NO, Krecek RC, Taner-Mulla D, Verweij JJ, Levecke B, Tannich E, Clark CG, Stensvold CR: Diversity and distribution of Blastocystis sp. subtypes in non-human primates. Parasitology 2013, 140(8):966-971.
26. Yoshikawa $\mathrm{H}$, Abe N, Wu Z: PCR-based identification of zoonotic isolates of Blastocystis from mammals and birds. Microbiology 2004, 150(Pt 5):1147-1151.

27. Stensvold CR, Alfellani M, Clark CG: Levels of genetic diversity vary dramatically between Blastocystis subtypes. Infect Genet Evol 2012, 12(2):263-273

doi:10.1186/1756-3305-6-215

Cite this article as: Wang et al.: Diversity of Blastocystis subtypes in dogs in different geographical settings. Parasites \& Vectors 2013 6:215.

\section{Submit your next manuscript to BioMed Central and take full advantage of:}

- Convenient online submission

- Thorough peer review

- No space constraints or color figure charges

- Immediate publication on acceptance

- Inclusion in PubMed, CAS, Scopus and Google Scholar

- Research which is freely available for redistribution

Submit your manuscript at www.biomedcentral.com/submit
Ciomed Central 